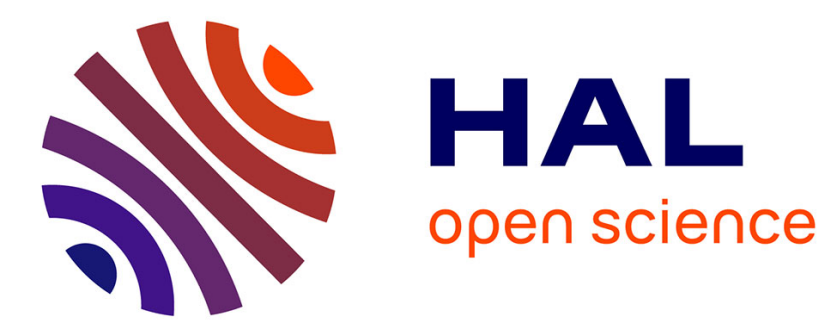

\title{
Prediction and Measurement of Sealing Properties of Joints Between Wavy Metal Surfaces
} Julien Bourniquel, Didier Lasseux, Jean-François Rit

\section{To cite this version:}

Julien Bourniquel, Didier Lasseux, Jean-François Rit. Prediction and Measurement of Sealing Properties of Joints Between Wavy Metal Surfaces. Journal of Pressure Vessel Technology, 2018, 140 (5), 10.1115/1.4040894 . hal-02367301

\section{HAL Id: hal-02367301 \\ https://hal.science/hal-02367301}

Submitted on 17 Nov 2019

HAL is a multi-disciplinary open access archive for the deposit and dissemination of scientific research documents, whether they are published or not. The documents may come from teaching and research institutions in France or abroad, or from public or private research centers.
L'archive ouverte pluridisciplinaire $\mathbf{H A L}$, est destinée au dépôt et à la diffusion de documents scientifiques de niveau recherche, publiés ou non, émanant des établissements d'enseignement et de recherche français ou étrangers, des laboratoires publics ou privés. 


\title{
Prediction and measurement of sealing properties of joints between wavy metal surfaces
}

\author{
Julien Bourniquel \\ CNRS, I2M, UMR5295 \\ Esplanade des Arts et Métiers \\ 33405 TALENCE Cedex France \\ EDF R\&D \\ Avenue des Renardières - Ecuelles \\ 77818 MORET-SUR-LOING Cedex France
}

\author{
Didier Lasseux * \\ CNRS, I2M, UMR5295 \\ Esplanade des Arts et Métiers \\ 33405 TALENCE Cedex France
}

\author{
Jean-Francois Rit \\ EDF R\&D \\ Avenue des Renardières - Ecuelles \\ 77818 MORET-SUR-LOING Cedex France
}

\begin{abstract}
The transmissivity of metal-metal sealing joints is investigated experimentally and compared to predictions obtained by modelling. The focus is laid upon a wavy surface contacting a flat rigid part, representative of a seat-to-plug contact in an internal sealing valve encountered in nuclear power plants for instance.

Experimental transmissivities are obtained from water leak-rate and pressure drop measurements carried out on a model ring-shape sample seat holding a controlled wavy defect and pressed against a rigid flat plug with a controlled normal load. The sample seat surface is manufactured by face turning a tubular part under radial stress and waviness is obtained after elastic relaxation.

Modelling is performed on a 3D finite element model of the assembly, composed of the plug, the sample seat and its holder. The upper sample seat surface, which topography is recorded by confocal microscopy, is reconstructed using a modal decomposition on a basis of vibrational eigen modes. Its lower surface, in contact with the holder, is considered as perfectly flat or with its own defects. The contact aperture field between the seat and the plug is computed for a given normal load and is used to solve the incompressible Reynolds equation with a boundary element method, yielding the transmissivity.

Predicted transmissivities reveal to be in good agreement with experimental data at low clamping loads and are overestimated for larger ones. Defects on the lower surface of the seat are shown to have a significant impact on the seat-to plug contact transmissivity.
\end{abstract}

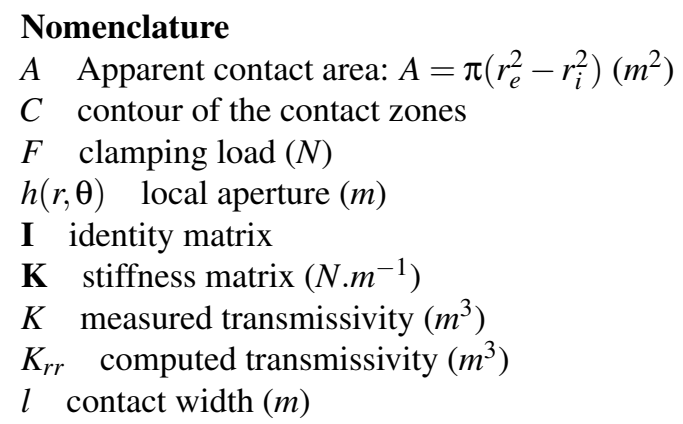

${ }^{*}$ Corresponding author. Email: didier.lasseux@u-bordeaux.fr 
$L$ contact length $(m)$

M matrix of mass $(\mathrm{kg})$

n unit normal vector to $C$ directed from the fluid-phase to the solid-phase

$P c a \quad$ Apparent contact pressure: $P c a=\frac{F}{A}(P a)$

$p_{\text {int }} \quad$ upstream pressure $(\mathrm{Pa})$

$p_{\text {ext }} \quad$ downstream pressure $(\mathrm{Pa})$

$\mathbf{q}_{v}$ local vector of flow rate per unit width $\left(m^{2} . s^{-1}\right)$

$Q$ macroscopic volume flow rate $\left(m^{3} \cdot s^{-1}\right)$

$r_{i}$ internal contact radius $(m)$

$r_{e}$ external contact radius $(m)$

$t$ time $(s)$

u displacement vector $(m)$

$\mathbf{U}_{i} \quad i^{\text {th }}$ eigenvector

\section{Greek letters}

$\Delta P$ pressure drop between the upstream and downstream regions of the contact $\Delta P=p_{\text {int }}-p_{\text {ext }}(P a)$

$\mu$ dynamic viscosity (Pa.s)

$\omega_{i} \quad i^{t h}$ angular frequency $(H z)$

$\theta$ azimuthal angle $(\mathrm{rad})$

\section{Introduction}

Direct contact between metal surfaces is sometimes necessary in order to ensure static sealing. It is of major interest in many industrial domains whenever severe temperature and pressure conditions prohibit the use of a polymer seal. This situation is often encountered in the nuclear industry where temperature and pressure exceed 280 degree Celsius and 160bar respectively. The present work is focused on the study of liquid water flow through plane annular metal joints of nuclear valves. Indeed, operating pressurized water reactors in nuclear power plants relies on sectioning valves of many sizes and types. In a closed valve, internal sealing, namely the capability of containing the leak-rate between a high upstream pressure and a low downstream pressure, is achieved by pressing a moving plug against a seat (see Fig. 1a). Repeated operations require hard alloy coating and lapping in order to enhance sealing properties. However, it has been demonstrated that the seat surface can be subject to wavy deformations induced by the manufacturing process and by thermal shocks during the device lifetime [1]. These surface distortions can induce sealing failure (Fig. 1b). The aim of this article is to evaluate the sealing efficiency of such a contact between a model wavy distorted seat and a plug using experiments and numerical predictions based on simulations of the deformation of the seat surface and fluid flow through the contact.

Sealing of metal joints contact is a difficult problem to simulate due to the multiscale character of surface defects on the one hand and the multiphysical aspects governing the leak-rate on the other hand. Indeed, defects can range from submicronic scale (roughness) to the whole contact scale (wavy or form defects) while computation of the leak-rate through the metal contact requires a combination of contact mechanics and fluid flow simulations. Nevertheless, no fluid/structure interaction is usually expected since fluid pressure remains small compared to the contact pressure between surfaces. Since flow within the contact occurs at low Reynolds numbers and because the interstitial aperture is slowly varying, the Reynolds (or lubrication) approximation is employed yielding two-dimension flow equations [2]. Reynolds approximation is widely used in the literature, in particular for lubrication problems [3,4]. Static sealing remains a particular case where contact surfaces do not move with respect to each other.

Each scale of defect, from roughness to waviness, combined with contact load, may impact the flow mechanism and strongly influence the leak-rate through the interstitial aperture field resulting from the contact. For face-turned surfaces, previous works have shown that leaking occurs predominantly in the radial direction, when contact load is sufficiently small, and switch to circumferential, through the main spiral groove, at large enough contact load. This was highlighted from results of numerical simulations $[2,5,6]$. It was further proven from direct measurements $[2,7,8]$ carried out on an experimental setup [9] designed for measurement of fluid flow-rate through a contact of rough metal surfaces. More recently, a direct in-situ observation of contact areas was reported [10], allowing the identification of radial and circumferential flow paths while increasing the clamping load.

An analysis focused on a hard alloy lapped surface without any significant wavy defect pressed against a plane sapphire, was carried out in a previous work. The dependence of the leak-rate to pressure drop relationship upon clamping load was investigated numerically considering the scale of roughness only [11,12]. Comparison with experimental results showed the difficulty of predicting accurately the sealing efficiency when one single scale of defects is taken into account [13]. While the heterogeneity of the material at the microscopic scale can not significantly modify the deformation of asperities and can hence not be put forth as a relevant explanation of the discrepancy between prediction and observation [14], it 


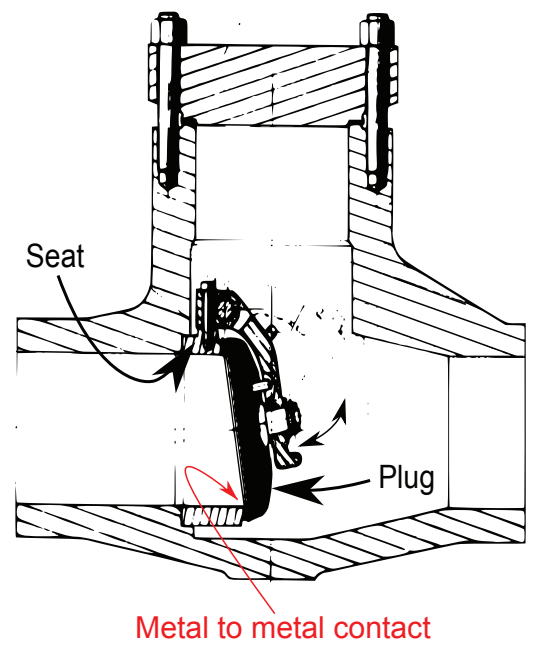

(a)

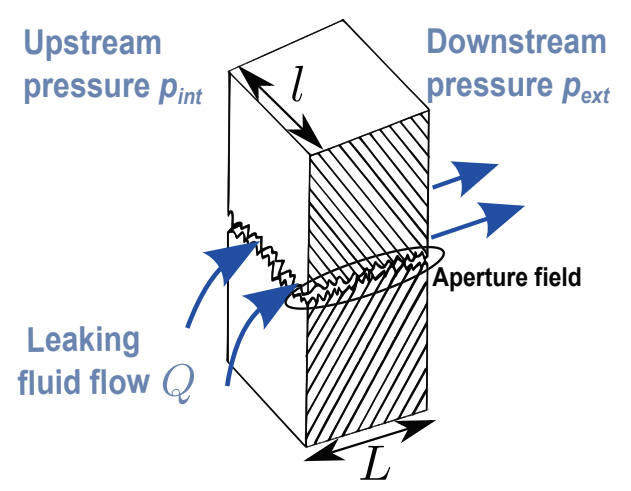

(b)

Fig. 1: Internal sealing

seems that the role of larger scales in the topological description of surface defects remains crucial for a realistic modeling of both deformation and flow. The impact of wavy defects on sealing efficiency was investigated numerically in a recent work [15] with an accent on the role of the different modes composing the defects as obtained from a modal decomposition (or vibrational analysis) [16]. However, no experimental results involving controlled wavy surfaces are available so far.

Under these circumstances, the aim of the present work is to carry out careful experiments to measure leak-rate versus clamping load using controlled model wavy surfaces in combination with simulations performed on their recorded topological representations for direct comparison without adjustable parameters. In order to leave as few uncontrolled parameters as possible, the sealing joint is isolated from the valve device as a separate system consisting of a flat and rigid plug and an annular seat holding a wavy defect. The wavy annular seat is manufactured by a process described in the experimental part of the paper. The numerical section of this paper provides a simulation model in order to predict the transmissivity which fully characterizes the contact for a given clamping load (see for instance $[2,12]$ ). To do so, a representation of the wavy surface of the seat is first derived from a modal decomposition $[16,17]$. Then, contact and deformation is simulated using the mechanical package code_aster [18]. Finally, Reynolds equations are solved within the resulting interstitial aperture field yielding the radial transmissivity versus the clamping load. The comparison between experimental and predicted transmissivities of the contact between the sample seat and the plug suggests that the finite rigidity of the seat combined with the defects on its opposite face in contact with the holder may significantly impact the contact properties with the plug, as confirmed by numerical investigations.

\section{Experimental setup and procedure}

The objective of the experimental part of the work is to perform accurate quantitative flow-rate measurement through the aperture field resulting from a controlled wavy defect on an annular surface (the seat) pressed against a flat plug. Such tightness tests and measurements could be performed on real valves. However, their interpretation would be difficult because of uncontrolled interactions between key parameters, such as fluid pressure and clamping load, as well as valve body deformations. As a consequence, a specific experimental setup was designed, focused on testing a model seat where every other parameter can be controlled (Fig. 2). Wavy deformation affecting the surface of the seat of a valve is typically about 50 micrometers in amplitude [1] and several millimeters in wavelength. This kind of defect is reproduced on the model seat using a specific manufacturing procedure. The flat rigid massive plug was made of tungsten carbide and is $56 \mathrm{~mm}$ in diameter. The lower surface was polished, yielding a measured planar defect of less than $0.3 \mu \mathrm{m}$ and a roughness $R a$ of $0.03 \mu \mathrm{m}$. Tolerancing on the plug was designed to be at least one order of magnitude more restrictive than what would be expected on a valve planar, lapped, seat surface, say a 30 $\mu \mathrm{m}$ planar defect and $R a \simeq 0.4 \mu \mathrm{m}$. Clamping of the contact between the model sample seat and the plug is servo-controlled and liquid water flow is forced by servo-controlling the flow rate through the contact while the corresponding pressure drop is recorded allowing the determination of the transmissivity as indicated below.

Before detailing the experimental procedure in sections 2.2 to 2.5 , the manufacturing process employed to generate a controlled wavy defect on the model seat is described in the following section. 


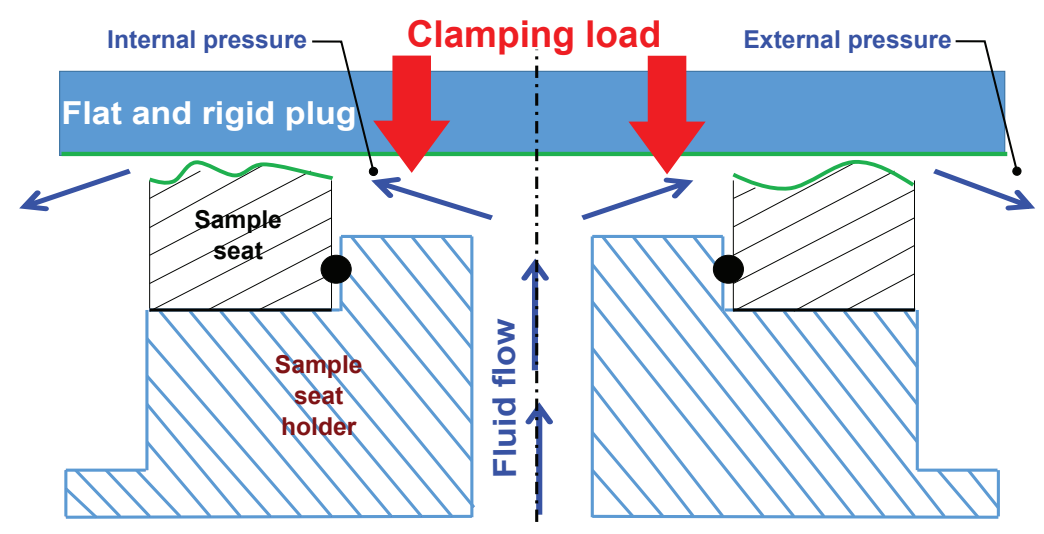

Fig. 2: Schematic representation of the sealing test device

\subsection{Wavy sample seat manufacturing}

The main idea to reproduce a controlled wavy defect on the model sample seat, is to manufacture the surface while the part is clamped, so that the wavy defect results from clamping release and elastic relaxation after machining. More specifically, the whole procedure includes the following seven steps.

1. A tubular part, which height and diameter are approximately equal, is machined by face turning and lapping (Fig. 3a), its top end being dedicated to the sample seat surface after machining. Materials characteristic of nuclear power plant valves are employed, namely 316L steel with Norem02 hard alloy coating [19] on the upper face.

2. The tubular part is clamped in a lathe's three-jaw chuck. Clamping load is chosen so as to achieve the final desired wavy defect amplitude. In order to reduce rigidity of the tubular part and favor its elastic deformation, three large axial slits are machined at the lower end of the part beforehand. Slits are placed in an alternate angular position with respect to the jaws in the chuck (see Fig. 3a).

3. While clamping load is maintained, the upper face of the part is flattened by face turning.

4. Lapping is operated on the plane surface of the sample seat in order to obtain a sealing surface finish, the part remaining clamped in the chuck. Both flattening and lapping operations reduce the initial $3.2 \mathrm{~mm}$ thickness of the hard coating by a negligible amount.

5. The tubular part is released from the chuck creating a wavy residual deformation on the upper surface due to elastic relaxation. This deformation is controlled using a needle tip dial indicator.

6. The upper part is cut using an electro-erosion technique in order to obtain the final model sample seat as illustrated in Fig. $3 b$.

7. Topology of the surface is recorded using confocal microscopy. An example for one of the sample seats (denoted Sample a) under concern in this work is reported in Fig. 4. These data are used as an input for the numerical simulation model that is described in section 3 dedicated to the numerical prediction.

Because of the three-jaw chuck clamping device, the ensuing wavy defect on the model sample seat surface has a threelobe structure. In real valves, the observed wavy defect, due to thermal shocks, rather exhibits two lobes [1]. In both cases wavy lobes are combined with a conical defect.

The model sample seat is mounted in the experimental setup for leak test and leak-rate measurements are carried out following the procedure described in the following section.

\subsection{Leak-rate measurement apparatus}

A specific experimental setup was designed ${ }^{1}$ to measure leak flow-rates in a large range of pressure and flow-rate conditions, namely up to $17.5 \mathrm{MPa}$ and $0.1 \mathrm{l} / \mathrm{s}$, with a maximal clamping load of $150 \mathrm{kN}$.

The flow-rate measurement system consists in servo-controlling two parameters to a nominal value: the plug to seat clamping load on the one hand, taking into account the upstream water pressure, and the liquid water flow through the interstitial aperture field on the other hand. Fluid flow can be controlled by either imposing the pressure drop or the flowrate. All the three measured values of pressure drop, clamping load and flow-rate are the necessary data to identify the value of the transmissivity. Constant flow-rate through the contact is ensured by three pistons associated in parallel which cyclic displacements are servo-controlled in a closed loop system (see Fig. 5). In addition, the whole experimental setup is

${ }^{1}$ LF Technologies (Saint-Hilaire de Riez, France). 


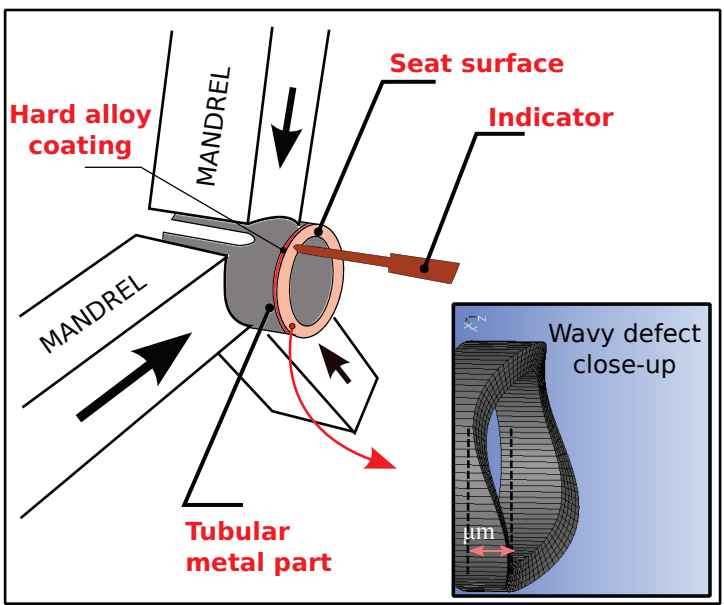

(a)

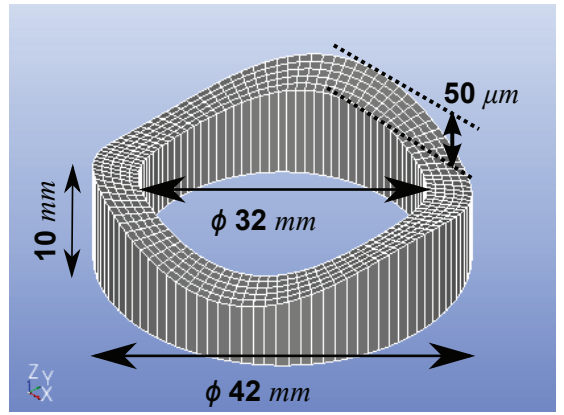

(b)

Fig. 3: Machining of the wavy defect on the sample seat

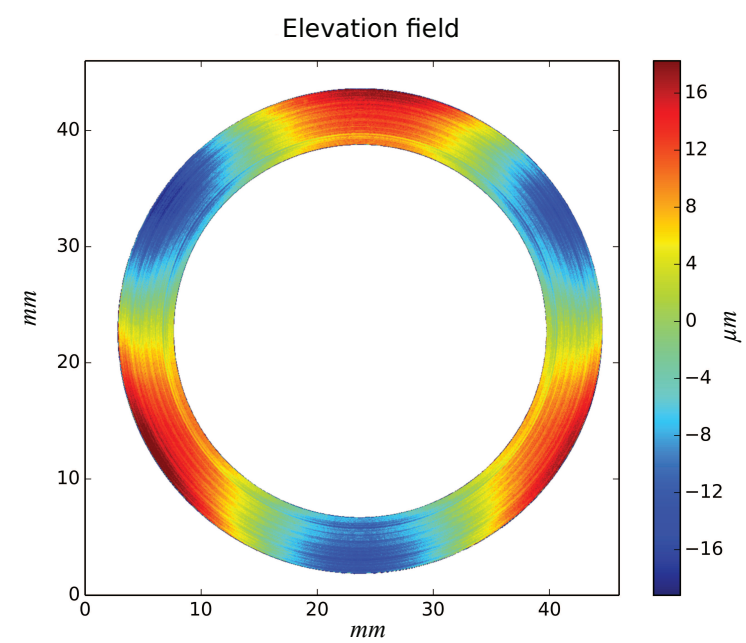

Fig. 4: Wavy defect measured by confocal microscopy. Sample a

temperature controlled with a separate liquid cooling circuit. Pressure drop is recorded by using two pressure sensors placed upstream and downstream of the contact, respectively.

A second experimental setup, differing in design from the first, was also used to cross-check measurements on the same sample seat and verify the relevance of the experimental apparatus above described. This alternative apparatus is based on the same principle of controlling the clamping force and flow through a stand-alone contact between a seat and a plug [9]. However, the plug is made of synthetic sapphire, water flow is imposed by a constant flow-rate syringe pump Isco 500D and mass flow-rate is measured at the outlet. Values of the transmissivity obtained with the two setups are compared in section 4 dedicated to the results.

\subsection{Determination of the transmissivity}

When flow occurs at a low Reynolds number, and because the slope of the wavy surface of the seat remains everywhere small compared to unity, the governing mass and momentum (Stokes) equations within the contact can be pre-integrated in the direction normal to the contact aperture field (see a derivation for instance in [12] and a more detailed one in [20]), yielding the Reynolds approximation. By denoting $Q, r_{i}, r_{e}, \Delta P=p_{\text {int }}-p_{\text {ext }}, \mu$ and $K$ the volume flow rate, internal and external contact radii, pressure drop, dynamic viscosity of the flowing fluid and transmissivity, the macroscopic Reynolds equation can be written under the form: 


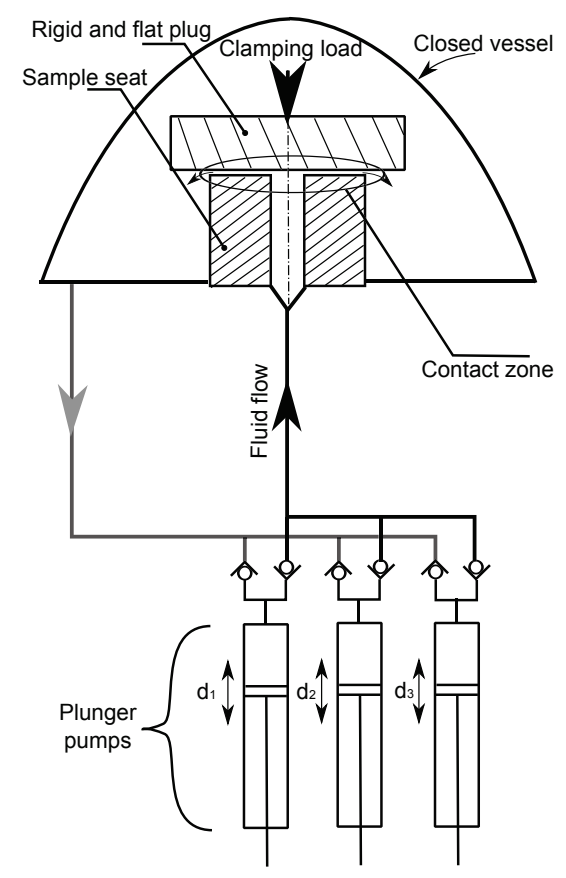

Fig. 5: Leak-rate measurement setup

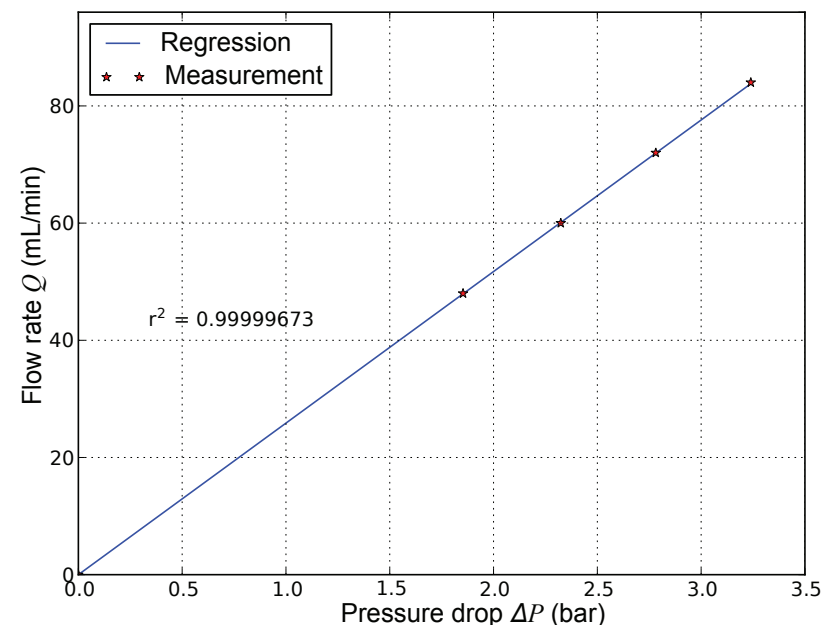

Fig. 6: Flow rate versus pressure drop for a clamping load of $12 \mathrm{kN}$ (i.e. an apparent contact pressure $P c a=20.7 M P a$ )

$$
\frac{Q}{2 \pi r_{i}}=-\frac{K}{\mu} \frac{\Delta P}{r_{i} \ln \left(\frac{r_{e}}{r_{i}}\right)}
$$

For a given clamping load (i.e. a given aperture field), transmissivity can be hence simply estimated from a linear regression on the $Q-\Delta P$ graph obtained from several measurement of flow-rates and corresponding pressure drops as illustrated in Fig. 6. Validity of the linearity assumption between pressure and flow-rate is checked directly for each transmissivity value. This procedure is repeated for several clamping loads, so that the dependence of $K$ on the apparent contact pressure, $P c a$, can be determined. The contact pressure is computed as the ratio of the clamping load, $F$, to the apparent contact area $A=\pi\left(r_{e}^{2}-r_{i}^{2}\right)$. Here, $r_{i}=16 \mathrm{~mm}$ and $r_{e}=21 \mathrm{~mm}$. Results presented hereafter were mainly obtained at small Reynolds number values although experimental data at large enough Reynolds numbers, featuring a non linear $Q-\Delta P$ relationship as a signature of significant inertial effects of the flow, will be shortly reported in section 2.5 . 


\subsection{Transmissivity and apparent contact pressure uncertainty}

Before describing the numerical procedure to compute the transmissivity, it is necessary to estimate the uncertainty on the determination of $K$ and $P c a$ from the experimental data. From Eqn. (1), global transmissivity uncertainty can be expressed as follows [7]:

$$
\frac{\Delta K}{K}=\frac{\Delta Q}{Q}+\frac{\Delta \mu}{\mu}+\frac{\Delta(\Delta P)}{\Delta P}+\frac{\Delta r}{\ln \left(\frac{r_{e}}{r_{i}}\right)}\left(\frac{1}{r_{e}}+\frac{1}{r_{i}}\right)
$$

In Eqn. (2), the term $\frac{\Delta \mu}{\mu}$ represents the relative error on the dynamic viscosity resulting from a temperature fluctuation. Typically, a fluctuation of about 0.5 degree Celsius can be observed during the experiment. This induces a $2 \%$ viscosity fluctuation. The error on $\Delta P$ results from pressure sensor accuracy and is equal to $0.072 b a r$ according to the device technical characteristics. Moreover, a fluctuation of $\Delta P$ induced by the servo-controlling device over the measurement period can reach $2 \%$ of the averaged value. The term $\frac{\Delta Q_{r}}{Q_{r}}$ corresponds to leak-rate error due to servo-controlling fluctuations which can be estimated to $3 \%$ as observed during initial tests carried out on the experimental setup. Finally, the error on the external and internal radii of the annular contact, $\Delta r$, can be estimated to $0.03 \mathrm{~mm}$. While a precise estimate of the total error on the determination of $K$ would require a more sophisticated calculation, we can simply consider cumulative errors as a first approach, keeping in mind that this represents an overestimate of the actual error. This leads to a relative error on $K$ that does not exceed $17.5 \%$.

For $P c a$, a similar approach yields

$$
\frac{\Delta P c a}{P c a}=\frac{\Delta F}{F}+\frac{2 \Delta r}{r_{e}-r_{i}}
$$

In Eqn. (3), $\frac{\Delta F}{F}$ is taken to $1 \%$ according to the technical characteristics of the load cell. Consequently, the apparent contact pressure uncertainty can be estimated to $2.2 \%$.

\subsection{Validity of the $Q-\Delta P$ linear relationship}

In order to check the validity of the low Reynolds number assumption which conditions the linear dependence of the flow-rate to pressure drop relationship, an experiment was carried out over a wide range of pressure drop, from 15bar to 170bar. This was performed for $P c a=50 M P a$ and the corresponding experimental results are reported in Fig. 7. One can observe that linearity persists for a pressure drop up to about 60bar, a range of $\Delta P$ where inertial effects are obviously negligible. Above this value, the non-linearity due to inertia indicates that the leak-rate can not be characterized by the transmissivity only and that a corrective term must be considered. More investigation is necessary to determine whether the pressure drop correction term is rather cubic or quadratic in flow-rate. This point is beyond the scope of this work and will not be further discussed here.

\section{Numerical prediction}

Numerical estimation of the transmissivity as a function of the normal clamping load is performed in this section through a set of mechanical simulations. This is carried out following a procedure divided into three steps (see a schematic representation in Fig. 8). It consists first in a model construction of the wavy defects (for the upper surface or for both the upper and lower surfaces; see below) as measured from confocal microscopy, an example of which is provided in Fig. 4 above. The synthetic reconstruction of the wavy surface is achieved by using the modal discrete decomposition method [15, 16]. It allows the extraction of the defects which scales range from that of wavines to much lower ones such as roughness for instance [17]. Regarding the contact between the sample seat and the holder, two different approaches are followed. The first one consists in assuming that both surfaces are free of defects, yielding a perfectly flat and complete contact. However, topographic records on the lower surface of the sample seats indicate that the typical amplitude of defects on this surface is about 15 to $20 \mu \mathrm{m}$ whereas it remains smaller than about $6 \mu \mathrm{m}$ on the upper surface of the holder. For these reasons, a second approach was followed which consists in taking into account defects on the sample seat lower surface, keeping the upper surface of the sample seat holder as perfectly flat. Once the reconstructed wavy surface(s) and bodies of the sample seat and holder are discretized, contact mechanics between the rigid flat plug and the wavy sample seat can be computed for a given apparent contact pressure, yielding the aperture field. This is performed using the mechanical package code_aster. Finally, fluid flow is computed through the resulting interstitial aperture field. This yields the transmissivity for the applied clamping load which can be directly compared to experimental results obtained from the procedure detailed in the previous section. Results obtained with the two above mentioned modeling approaches are reported in sections 4.1 and 4.2 respectively. 


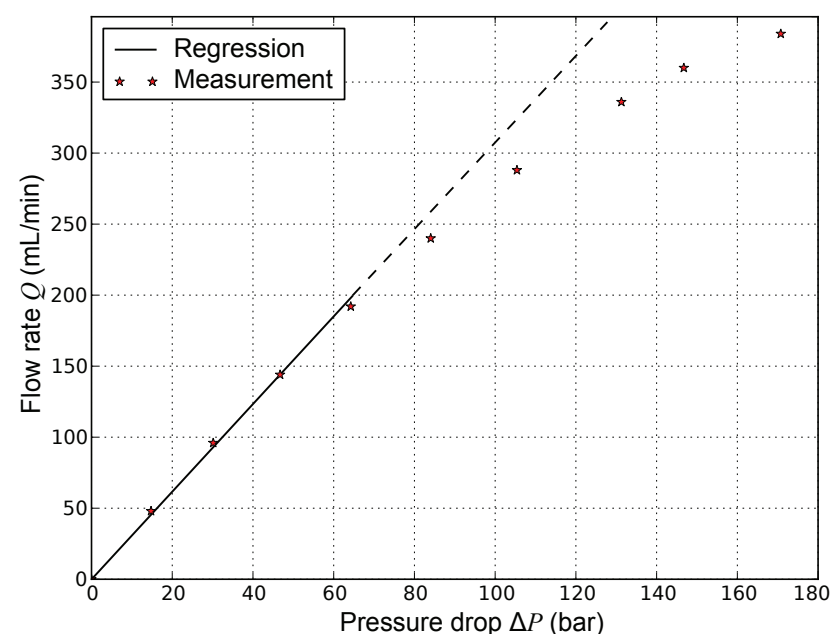

Fig. 7: Domain of validity of the low Reynolds approximation for $P c a=50 M P a$

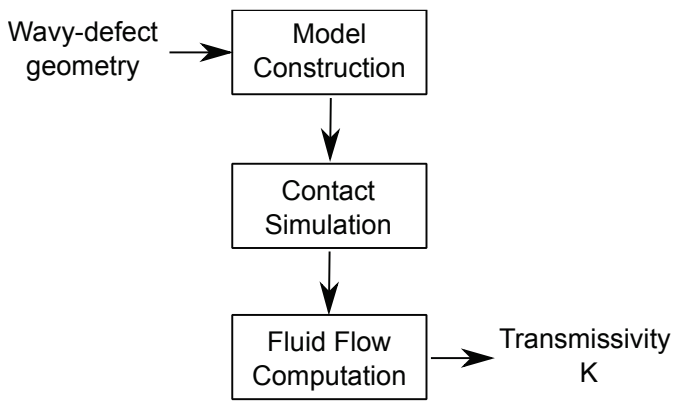

Fig. 8: Simulation steps

\subsection{Model defect construction}

The first step of the simulation procedure is to provide a relevant model of the wavy defect imprinted on the model sample seat used in the experiment. Here, it is assumed that leakage is mainly governed by waviness and, consequently, lower scale defects are filtered out from the model. To accomplish this task, the modal discrete decomposition method is employed. This method uses vibrational modes of a flat annular surface in order to provide a geometric basis of defects [15, 16]. More precisely, the method is applied on a ring surface of $16 \mathrm{~mm}$ and $21 \mathrm{~mm}$ in inner and outer radii, respectively, corresponding to the annular contact under study.

The geometric defect basis is built with the eigenvectors displacement modes obtained from the solution of the dynamic conservative equilibrium given by:

$$
\mathbf{M} \cdot \frac{\partial^{2} \mathbf{u}}{\partial t^{2}}+\mathbf{K} \cdot \mathbf{u}=0
$$

In this equation, $\mathbf{M}$ and $\mathbf{K}$ are the mass and stiffness matrices, respectively, while $\mathbf{u}$ is the displacement vector and $t$ the time variable. Determination of the matrices and resolution is carried out using the mechanical package code_aster. In this study, $\mathbf{u}$ is restricted to node displacements along the ring axis.

The displacement eigenvectors, $\mathbf{U}_{\mathbf{i}}$, are obtained from the solution of the linear system resulting from the discrete form of Eqn. (4) and are normalized in the sense of the infinity norm in order to express modal decomposition coefficients in a consistent unit. Each natural distortion mode is then associated to an angular frequency $\omega_{i}$ according to:

$$
\left(\mathbf{M}^{-1} \cdot \mathbf{K}-\frac{1}{\omega_{i}^{2}} \mathbf{I}\right) \cdot \mathbf{U}_{\mathbf{i}}=0
$$

where $\mathbf{I}$ is the identity matrix. 


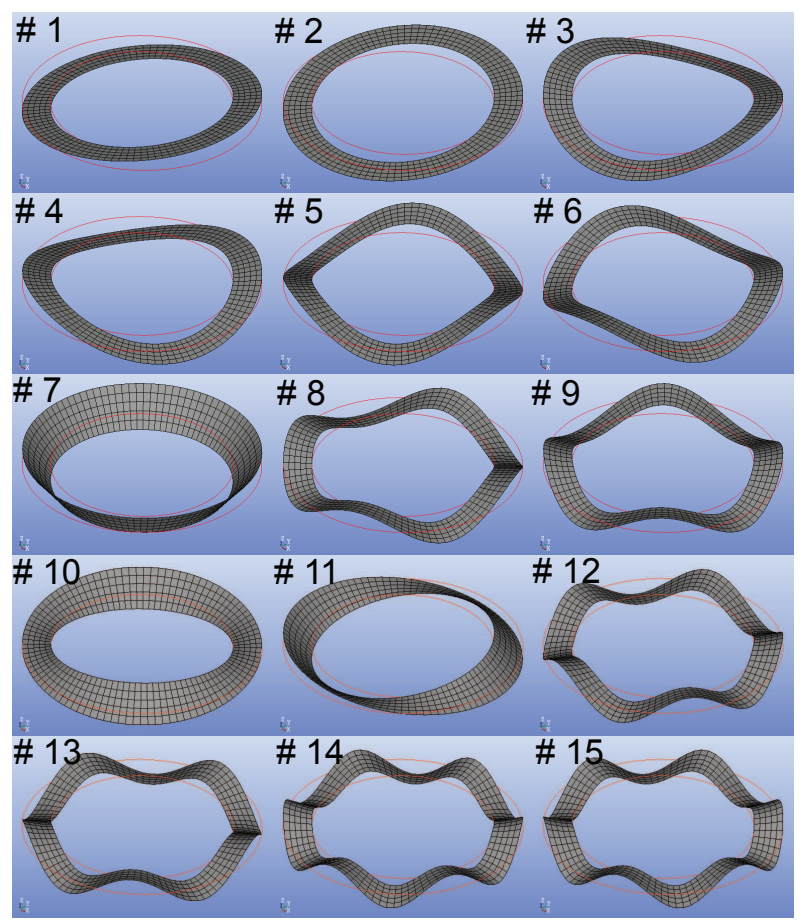

Fig. 9: Geometry of the first fifteen defect modes

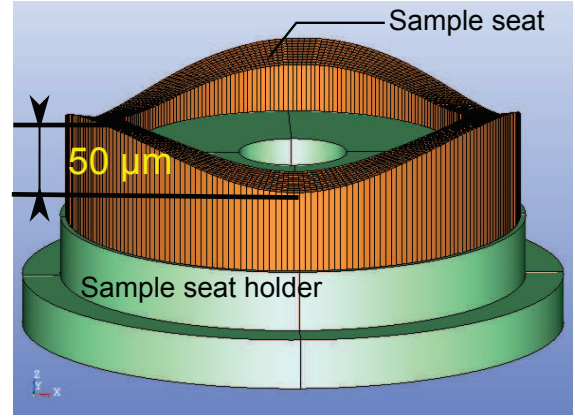

(a)

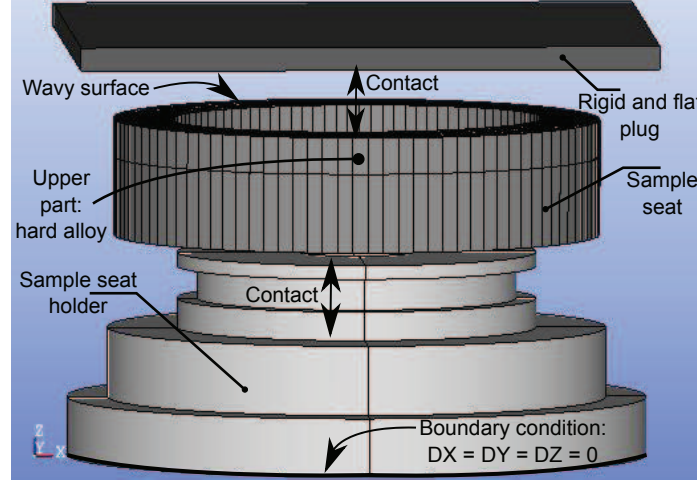

(b)

Fig. 10: Model construction

The advantage of this method lies in the fact that it is exhaustive while its scope is general as it can be applied to any kind of surface holding any kind of wavy defect [15]. Moreover, with this method, the eigenvectors, which define the decomposition basis, can be sorted according to their corresponding increasing angular frequency, so that modes of increasing complexities can be identified on the surface. This basis, which is theoretically infinite, can be truncated at the desired order. Here, the 25 first natural modes were found appropriate to accurately reproduce the wavy defect observed on the real surfaces by confocal microscopy. The first fifteen modes obtained for the upper annular surface of Sample a) are represented in Fig. 9. The wavy defect measured by confocal microscopy is hence decomposed on the modal basis of the first 25 modes $\left(U_{i}\right)_{i=1,25}$. It is used in the model representing the assembly within the experimental setup as shown in Fig. $10 \mathrm{a}$ where the amplitude of the wavy defect was amplified for clarity. The same methodology is employed while taking into account the defect on the lower surface of the sample as will be reported in section 4.2. The following step is the contact simulation between the constructed model and the flat rigid surface of the plug.

\subsection{Contact simulation}

The whole contact model implemented in code_aster is represented in Fig. 10b. The 3D model includes the sample seat positioned on the holder and the flat rigid plug. Both contacts between the seat and the holder and between the wavy 


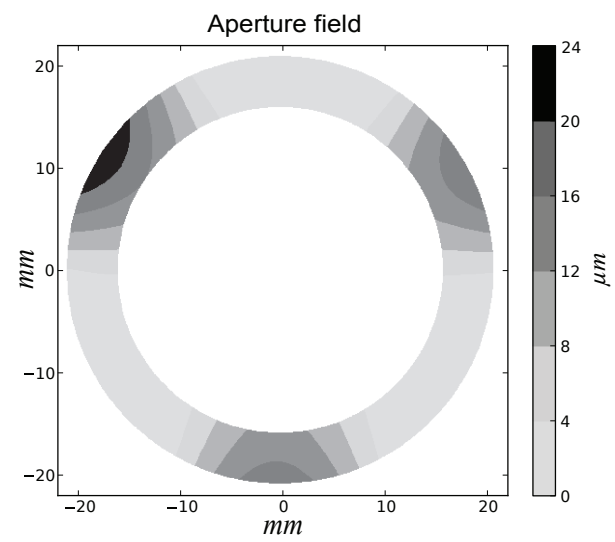

Fig. 11: Aperture field between the upper wavy surface of the sample seat and the plug resulting from contact simulation with an apparent contact pressure $P c a=15.5 \mathrm{MPa}$. Sample a

surface and the plug are part of the simulation. A zero displacement boundary condition is imposed at the lower face of the holder. Material constitutive laws are implemented as reported in previous studies [19]. In particular, the seat, which is $10 \mathrm{~mm}$ in thickness, is modeled as a $3.2 \mathrm{~mm}$ hard alloy layer at the top, the remaining being made of $316 \mathrm{~L}$ steel. The plug is considered as an infinitely rigid body while the holder is made of F16PH steel. The simulation is carried out by imposing the plug displacement. For each displacement step, clamping load $F$ is computed from the contact pressure integral over the effective contact area yielding the apparent contact pressure $P c a=\frac{F}{A}$. In addition, the aperture field, $h(r, \theta)\left(r_{i} \leq r \leq r_{e}\right.$, $0 \leq \theta \leq 2 \pi$ ), computed as a result of the wavy surface deformation, is recorded for further use in the flow computation to determine the transmissivity. An aperture field example obtained after modal decomposition of the recorded manufactured wavy surface of Sample $a$ is represented in Fig. 11 for $P c a=15.5 \mathrm{MPa}$, clearly highlighting the signature of the three-lobe and conical structure. This result was obtained while assuming perfectly planar contact surfaces between the sample seat and the holder.

\subsection{Fluid flow computation}

Fluid flow is computed within the interstitial aperture field $h(r, \theta)$ assuming no inertial effects and adopting the Reynolds model which reduces the problem to $2 \mathrm{D}$, in the plane of the contact, so that the governing equations are given by $[12,20]$ :

$$
\begin{gathered}
\mathbf{q}_{\mathbf{v}}=-\frac{h^{3}}{12 \mu} \nabla p \\
\nabla \cdot \mathbf{q}_{\mathbf{v}}=0 \\
\mathbf{q}_{\mathbf{v}} \cdot \mathbf{n}=0 \quad \text { on } C \\
p=p_{\text {int }} \quad \text { at } r=r_{i} \\
p=p_{\text {ext }} \quad \text { at } r=r_{e}
\end{gathered}
$$


Here, $\mathbf{q}_{\mathbf{v}}$ is the local flow-rate per unit width, $C$ is the contour of the contact zones and $\mathbf{n}$ is the unit normal vector to $C$ pointing out of the fluid phase while $p_{\text {int }}$ is the internal pressure $\left(p_{\text {int }}>p_{\text {ext }}\right), p_{\text {ext }}$ remaining equal to the atmospheric pressure.

Equations (6) to (10) refer to a diffusive problem for which local transmissivities, $\frac{h(r, \theta)^{3}}{12}$, form an heterogeneous field $[12,15]$ which is obtained from contact simulation. The computation of the pressure and local fluid flow $\left(p, \mathbf{q}_{\mathbf{v}}\right)$, solution of the above boundary value problem, is carried out with a numerical code based on an integral formulation of Eqns. (6) to (10) and a boundary element method (see Chap. 7 in [21]). The leak-rate $Q$ is computed from an integral of $q_{v}$ at $r=r_{i}$ ( or $r=r_{e}$ ). Transmissivity of the contact is deduced from the averaged Reynolds equation [12], as already used to interpret experimental results (see Eqn. (1)), and is given by:

$$
K_{r r}=\frac{\mu Q \ln \left(\frac{r_{e}}{r_{i}}\right)}{2 \pi\left(p_{\text {int }}-p_{\text {ext }}\right)}
$$

Transmissivity is computed for several aperture fields corresponding to apparent contact pressures ranging from 1 to around $70 \mathrm{MPa}$ which represent typical values encountered in practical use of valves in nuclear power plants. A direct comparison of $K_{r r}$ with $K$ obtained from the experiments described above can then be performed.

\section{Results and discussion}

\subsection{Contact with a zero defect assumption between the sample seat and the holder}

In Fig. 12, we have represented the dependence of the transmissivity upon the apparent contact pressure $P c a$, as determined experimentally and estimated from the computational procedure described above, while considering that the contact between the sample seat and the holder is free of defects. These results were obtained for three different samples denoted Sample $a$, Sample $b$ and Sample $c$. The relative difference between $K$ and $K_{r r}$, taking $K$ as the reference value, are also represented in Fig. 12. The prediction remains relatively precise for apparent contact pressures smaller than $10 M P a$. It is excellent in the case of Sample $a$ while an overestimation by a factor of roughly 1.5 to 1.75 can be observed for Sample $b$ and Sample $c$ respectively. For larger values of $P c a$, the transmissivity is overestimated by a factor that can reach roughly 3.5 .

The experimental relative error on $K$, which was (over)estimated to be $17.5 \%$ at most in the experimental section above, can obviously not explain this discrepancy with the computed values reported in Fig. 12. Moreover, transmissivities determined from measurements carried out on the two different experimental devices presented in section 2.2 are in excellent agreement as indicated in Fig. 13. This can be clearly seen from the relative difference between the values of $K$ obtained with apparatus 1 (taken as the reference) and 2 also reported in Fig. 13. The two measurements of $K$ differ by $10 \%$ at most over the whole range of Pca. This confirms that no experimental artifact is expected in these results. Experiments were all performed in the regime where an excellent linear relationship between the flow-rate and the pressure drop was observed, justifying the use of Eqn. (1) to interpret them.

\subsection{Impact of defects on the sample seat lower surface}

A careful attention to the defects on the lower surface of the sample seat in contact with the holder indicates that their amplitude is not negligible compared to that of waviness on the upper surface for all the three samples used in the experiments. As shown in Fig. 14, where the elevation fields of the upper and lower surfaces of each sample were reported, the defect amplitude on the lower surface is about 50\% (respectively $40 \%$ and 30\%) of that on the upper surface for Sample a (respectively Sample $b$ and Sample c). Since the rigidity of the sample seat is not infinite, it is of prime interest to investigate the impact of this defect on the aperture field on the upper contact with the plug resulting from deformation. As a first approximation, the surface of the holder is considered perfectly flat. This is motivated by the fact that the defect on this surface remains smaller than $5 \mu m$ in amplitude.

Transmissivity results obtained from simulations where defects are present in the contact surface between the seat and the holder are reported in Fig. 15 for comparison with the preceding model (i.e. assuming a perfect contact) and experimental results. Clearly, the impact of the lower contact configuration on the transmissivity is significant and can not be ignored in the modelling. For both Sample $b$ and Sample $c$, the prediction of $K$ is improved although an opposite conclusion holds for Sample a.

Although significant, this effect can not completely explain the difference between the predicted and measured values of the transmissivity. It should be noted that, in both approaches, the numerical estimation of the transmissivity relies on a major assumption that deformation and fluid flow can be modelled by considering wavy defects only. This is based on the idea that their amplitude is such that their impact must be dominant in the flow process. On this basis, roughness was filtered out from 


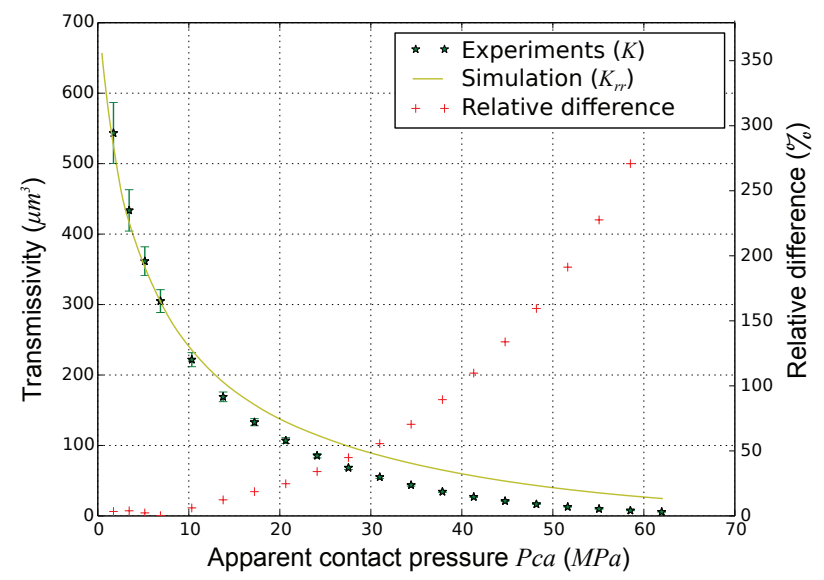

(a)

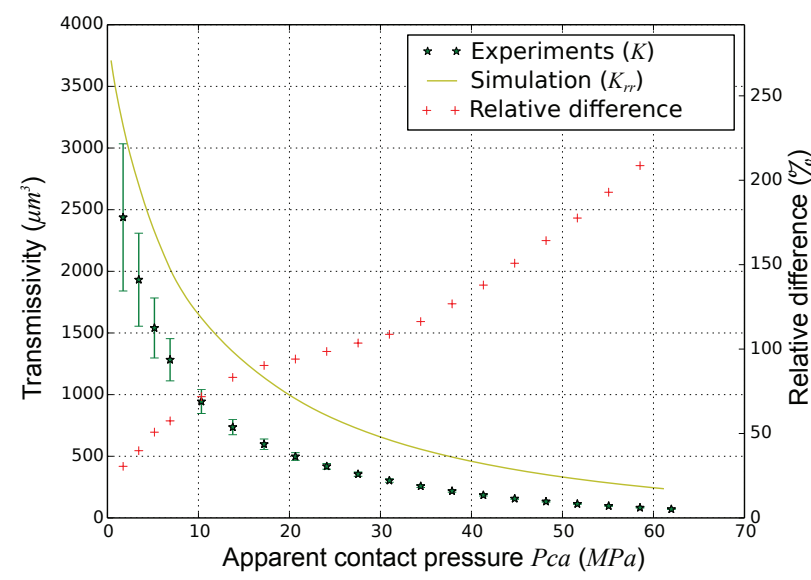

(b)

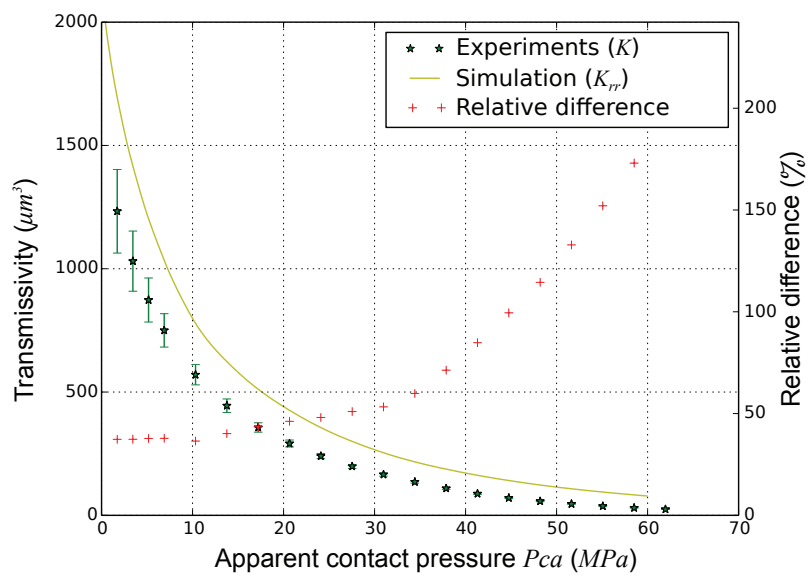

(c)

Fig. 12: Comparison between numerical prediction and experiments. a) Sample a. b) Sample b. c) Sample c. The relative difference is computed using the experimental values as the reference

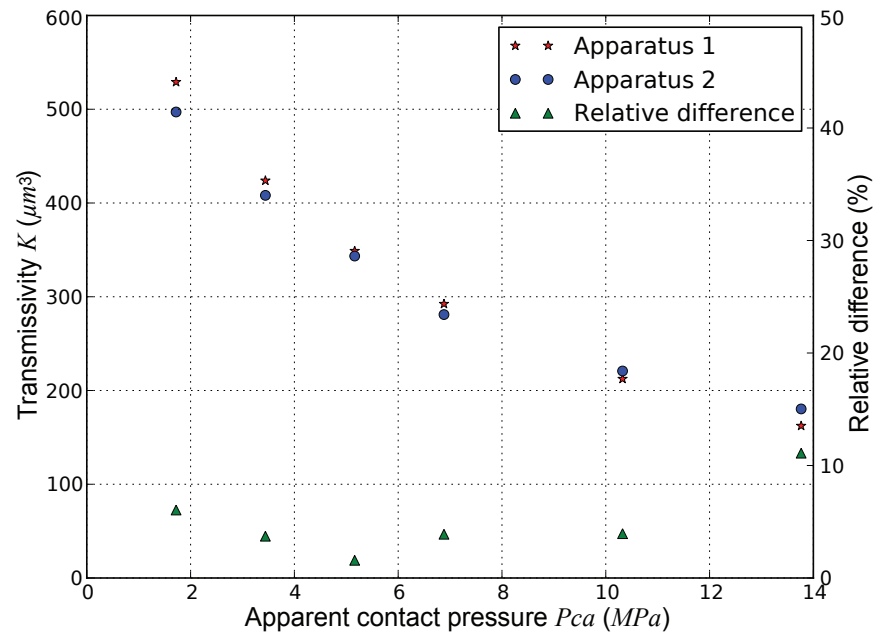

Fig. 13: Transmissivities obtained from measurements performed on apparatus 1 (see schematic representation in Fig. 5) and 2 (see [9] for details). Transmissivity obtained with apparatus 1 is used as the reference for the \% relative error 
the representation of the sample seat upper surface while employing the modal decomposition. This was further motivated by the fact that roughness, having a typical wavelength of about $10 \mu \mathrm{m}$ and an amplitude of 4 to $5 \mu \mathrm{m}$, would imply such a refined mesh size that the number of grid blocks required to take this defect into account would be absolutely prohibitive for a numerical simulation to be carried out, in particular regarding the computation of deformation. However, as evidenced by images of the manufactured wavy surfaces obtained by confocal microscopy, grooves are still present after lapping as a signature of the face turning process. These defects might have a negligible impact on the flow for small clamping load due to the fact that the surface is weakly deformed and waviness dominates roughness, explaining why the prediction of the transmissivity is in good agreement with experimental observation in this range of clamping load. However, with increasing load, deformation is more strongly affected by the existence of roughness, implying a significant modification of the aperture field and, as a consequence, of the transmissivity. Moreover, as load increases, waviness amplitude is progressively reduced and roughness in the valleys contributes to a significant increase of the pressure drop compared to a situation where this roughness is neglected. At this stage of the investigation, it is however still unclear what the quantitative contribution to deformation and flow these defects could have. Further work will be carried out to elucidate this multiscale effect that has been widely ignored so far.

\section{Conclusion}

In this paper, transmissivity of a plane contact under normal load, a property that fully characterizes the sealing efficiency of the assembly, is investigated from direct measurements and numerical simulations. The study is focused on the contact of a metallic machined surface holding a wavy defect pressed against a rigid and flat slab. The wavy defect is achieved from a specifically dedicated machining process with the purpose to mimic real contact surfaces, as those encountered in valves for instance. The industrial rationale for such an investigation is to obtain a quantitative predictive tool that relates leaking rates to observed or conjectured wavy defects, such as deformations occurring on valve seats subject to repeated thermal shocks.

Liquid flow rate through the annular contact is measured versus pressure drop and normal clamping load applied to the assembly. The linear relationship between the flow-rate and the pressure drop allows the determination of the transmissivity as a function of the apparent contact pressure, $\mathrm{Pca}$, to within less than $17.5 \%$ of error. These results are directly compared to the prediction of the transmissivity obtained from numerical simulations. The predictive tool relies on a model that takes into account the different parts of assembly present in the experimental device, namely the flat rigid surface in contact with the wavy surface of the sample and the sample holder. The lower surface of the sample contacting the holder is considered perfectly flat in a first step while the defects on this surface are taken into account in a second step. Topography of the wavy upper (and lower) surface(s) of the sample is recorded by confocal microscopy and further modelled using modal decomposition for implementation as an initial input in the numerical algorithm. The simulation consists in successively computing contact deformation using material constitutive laws in an elastic-plastic model and fluid flow using a boundary element method in order to compute the transmissivity for a given load applied to the assembly.

Direct comparison of experimental and computational results without any adjustable parameter shows that relatively accurate prediction for sufficiently small apparent contact pressures (less than $10 M P a$ ) is achieved whereas transmissivity is significantly overestimated for larger values of $P c a$. The difference between the measured and computed transmissivities is demonstrated to be very sensitive to the existence of defects in the contact between the sample and the holder. A significant improvement of the prediction with respect to the experimental observation is obtained for two of the three samples used in the experiments when these defects are taken into account in the numerical simulation. For one of the sample, this approach leads to an opposite tendency suggesting that the topography description of the surfaces is still incomplete for an accurate determination of the aperture field of interest. Nevertheless, this conclusion is a clear indication that modelling of contacts arranged in series requires special care when interest is focused on an accurate determination of the aperture field in one of these contacts as their complex interaction resulting from effort transmission might strongly modify the local deformation.

To improve the numerical model over the whole range of contact pressure, it seems necessary to take into account defects at much smaller scales than waviness. In particular, the existence of grooves as a remainder of the machining process might be of concern. Nevertheless, the accurate prediction at sufficiently small clamping loads which can be encountered, for example, in many situations involving check valves, opens wide perspectives for industrial applications and suggests to carry on improving the model for larger contact pressures.

\section{Acknowledgements}

Authors wish to acknowledge the contributions of J-P Mathieu, D. Hersant, C. Teygeman, S. Meunier, L. Aumond and S. Raposo-Mello from EDF Labs in designing the process to obtain samples featuring controlled wavy surfaces. 


\section{References}

[1] Mathieu, J.-P., Rit, J.-F., Ferrari, J., and Hersant, D., 2010. "Residual deformation of a hard-coated valve seat subjected to thermal shocks". In ASME 2010 Pressure Vessels and Piping Division/K-PVP Conference, American Society of Mechanical Engineers, pp. 211-217.

[2] Marie, C., Lasseux, D., Zahouani, H., and Sainsot, P., 2003. “An integrated approach to characterize liquid leakage through metal contact seal". European Journal Mech. and Env. Eng., 48(2), pp. 81-86.

[3] Prat, M., Plouraboué, F., and Letalleur, N., 2002. "Averaged Reynolds equation for flows between rough surfaces in sliding motion". Transp. Porous Media, 48(3), pp. 291-313.

[4] Plouraboué, F., Prat, M., and Letalleur, N., 2001. "Sliding lubricated anisotropic rough surfaces". Phys. Rev. E, 64, Jun, p. 011202.

[5] Geoffroy, S., and Prat, M., 2004. "On the leak trough a spiral-groove metallic static ring gasket”. J. Fluids Eng., 126, pp. 48-54.

[6] F. Robbe-Valloire, M. P., 2008. "A model for face-turned surface microgeometry application to the analysis of metmetal static seals". Wear, 264, pp. 980-989.

[7] Marie, C., 2002. "Fuite monophasique au travers d'un contact rugueux : contribution à l'étude de l'étanchéité statique". PhD thesis, Université Bordeaux 1.

[8] Marie, C., and Lasseux, D., 2007. "Experimental leak-rate measurement through a static metal seal”. J. Fluids Eng., 129(6), pp. 799-805.

[9] Lasseux, D., and Marie, C., 2008. Apparatus for measuring the amount of leakage of a sealed arrangement. Patent EP1336831.

[10] Nitta, I., Matsuzaki, Y., Tsukiyama, Y., Horita, M., and Sakamoto, S., 2013. "Thorough observation of real contact area of copper gasket using a laser microscope with a wide field of view". J. Tribol., 135, p. 041103.

[11] Vallet, C., Lasseux, D., Zahouani, H., and Sainsot, P., 2009. "Sampling effect on contact and transport properties between fractal surfaces". Tribol. Int., 42(8), pp. $1132-1145$.

[12] Vallet, C., Lasseux, D., Sainsot, P., and Zahouani, H., 2009. "Real versus synthesized fractal surfaces: Contact mechanics and transport properties". Tribol. Int., 42(2), pp. 250 - 259.

[13] Vallet, C., 2008. "Fuite liquide au travers d'un contact rugueux : application à l'étanchéité interne d'appareils de robinetterie". PhD thesis, Arts et Métiers ParisTech.

[14] Durand, J., Proudhon, H., and Cailletaud, J., 2012. "Contact between rough surfaces : crystal plasticity influence on the contact tightness estimation”. In Blucher Mechanical Engineering Proceedings, pp. 968-979.

[15] Ledoux, Y., Lasseux, D., Favreliere, H., Samper, S., and Grandjean, J., 2011. "On the dependence of static flat seal efficiency to surface defects". Int. J. Press. Vessels Pip., 88(11-12), pp. 518 - 529.

[16] Samper, S., and Formosa, F., 2007. "Form defects tolerancing by natural modes analysis". J. Comput. Inf. Sci. Eng., 7(1), pp. 44-51.

[17] LeGoic, G., 2012. "Qualité géométrique et aspect des surfaces : approches locales et globales”. PhD thesis, Université de Grenoble.

[18] EDF R\&D. Code_Aster, Structures and Thermomechanics Analysis for Studies and Research. http://www.codeaster.org.

[19] Beaurin, G., and Mathieu, J.-P., 2011. "Microstructural and mechanical properties evolutions of plasma transferred arc deposited norem02 hardfacing alloy at high temperature". Materials Science \& Engineering. A, 528(15), pp. 50965105.

[20] Zaouter, T., Lasseux, D., and Prat, M., 2018. "Gas slip flow in a fracture: local Reynolds equation and upscaled macroscopic model”. J. Fluid Mech., 837, pp. 413-442.

[21] Huyakorn, P., and Pinder, G., 1983. Computational methods in subsurface flow. Monograph Series. Academic Press. 


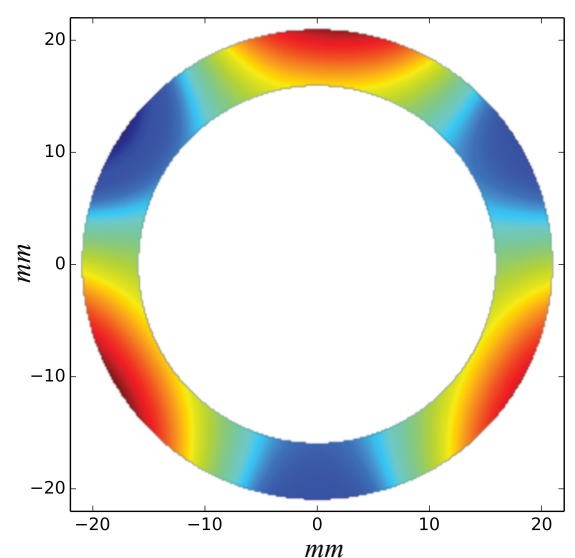

(a)

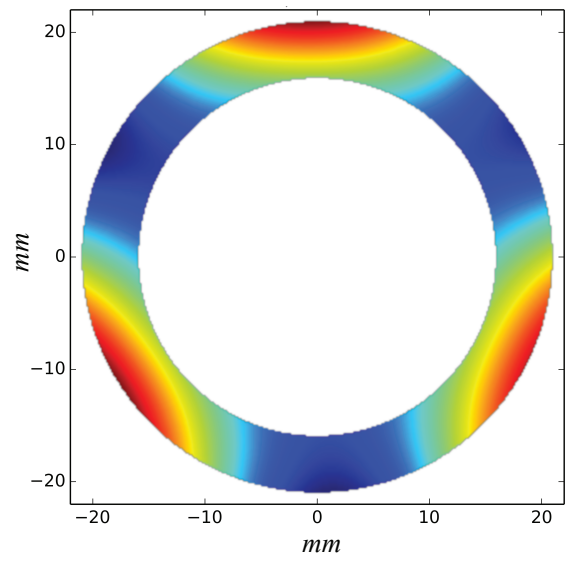

(c)

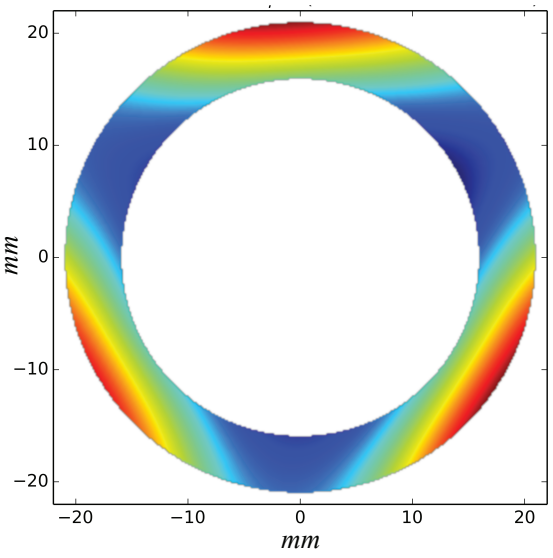

(e)

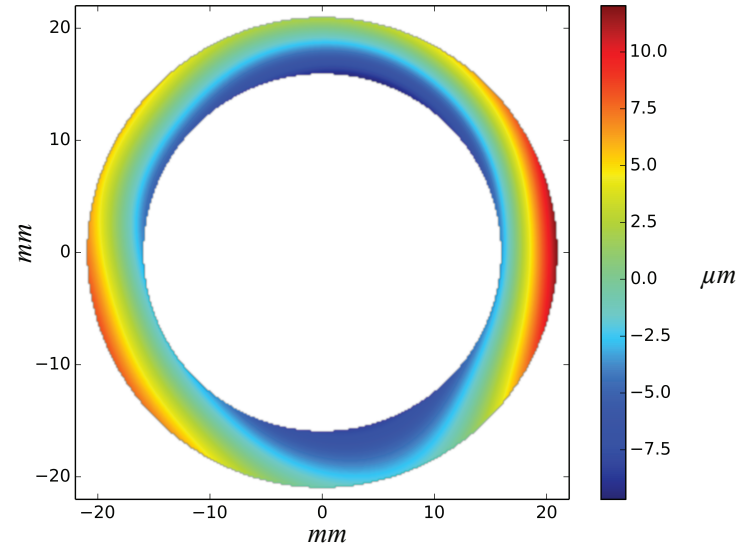

(b)

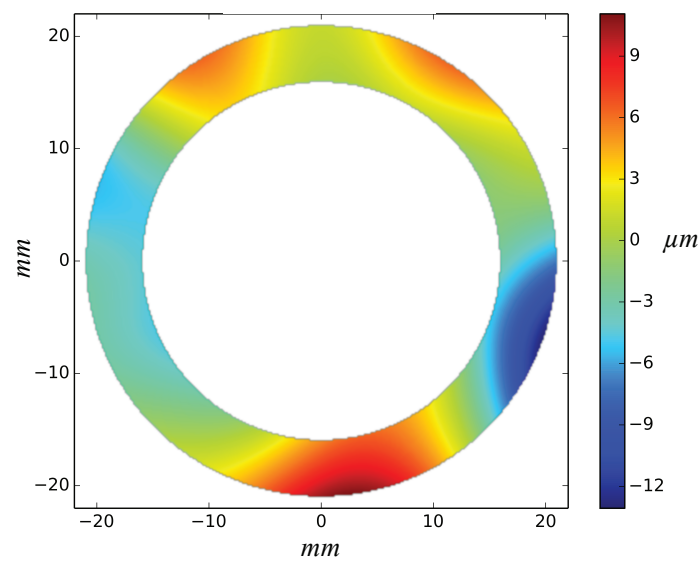

(d)

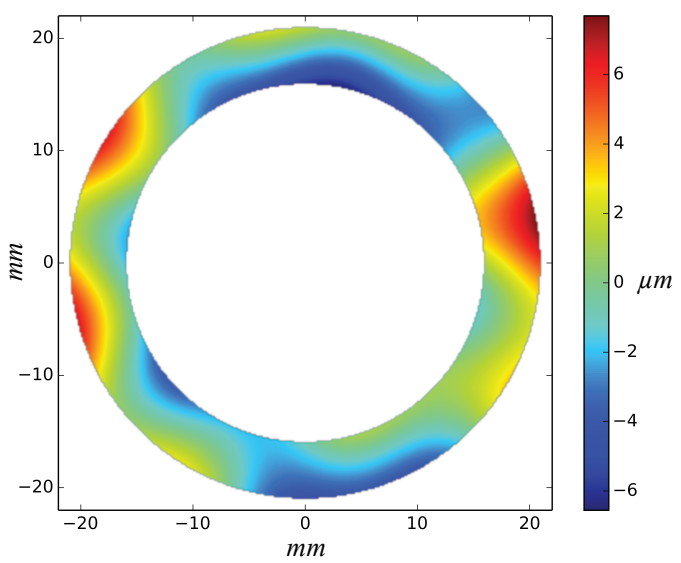

(f)

Fig. 14: Elevation fields on upper (left column) and lower (right column) surfaces of the sample seats used in the experiemnts. a)-b) Sample a. c)-d) Sample b. e)-f) Sample c 


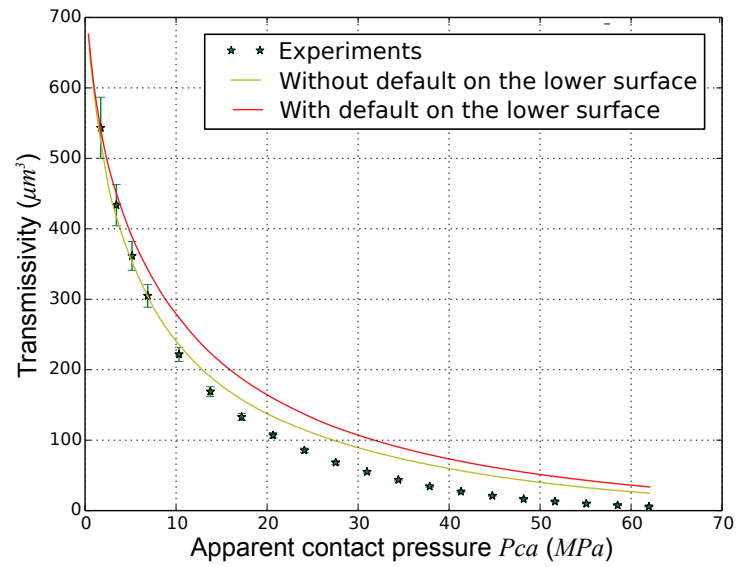

(a)

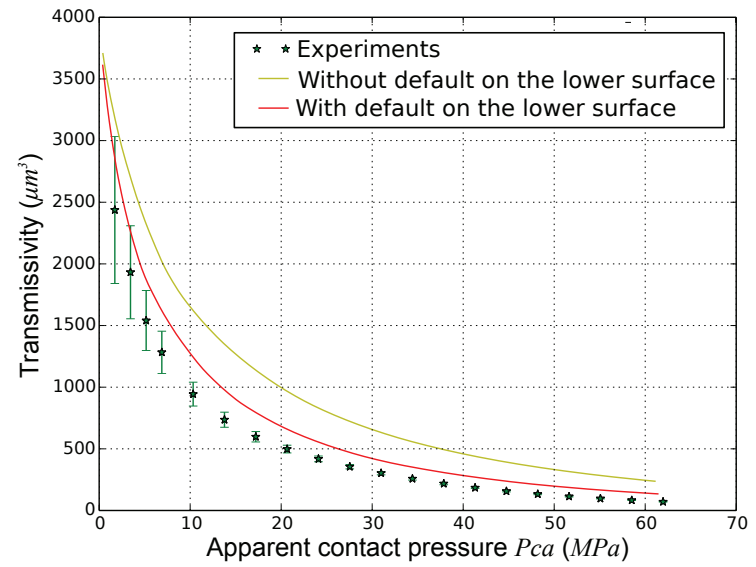

(b)

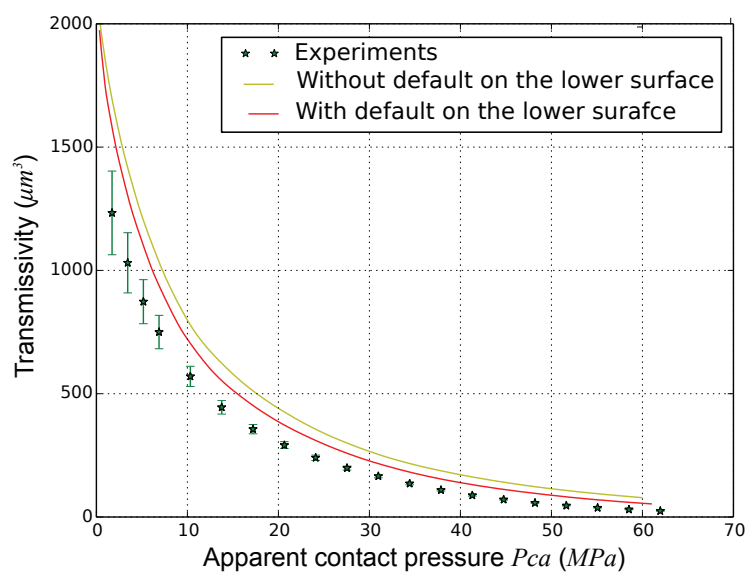

(c)

Fig. 15: Comparison of transmissivities obtained experimentally and numerically with and without defect on the lower surface of the sample seat. a) Sample a. b) Sample b. c) Sample c 